\title{
Virusbedingte Hepatitiden
}

$\mathrm{D}$ ie virusbedingte Hepatitis ist eine der weltweit häufigsten Infektionskrankheiten. Anders als die Hepatitis A und E, die so genannte Reisehepatitis, kann die Infektion mit den Hepatitisviren B oder C einen chronischen Verlauf nehmen. Mit etwa einer Million Patienten allein in Deutschland ist die chronische Virushepatitis mit ihren Folgen (Leberzirrhose oder ein hepatozelluläres Karzinom) ein großes gesundheitliches Problem. Daher ist es von besonderer Bedeutung, entsprechende Maßnahmen rechtzeitig einzuleiten und die neuesten Forschungsergebnisse in die Praxis umzusetzen. Um diese Aufgabe zu bewältigen, ist eine „vertikale“ und „horizontale“ Vernetzung zwischen Universitäten und niedergelassenen Ärzten bis hin zu den Patienten notwendig.

Genau diese Ziele verfolgt das Kompetenznetz Hepatitis (Hep-Net), das seit dem 1. Februar 2002 besteht und vom Bundesministerium für Bildung und Forschung (BMBF) gefördert wird. Die Struktur des Netzwerkes ermöglicht dabei, wichtige klinische Studien im Hep-Net „Study House“ mit neuen Therapiestrategien zur Behandlung der Hepatitis B und C durchzuführen. Bislang konnten mehr als 2000 Patienten im Study House behandelt werden. Damit Hep-Net auch nach der BMBFFörderphase eine optimale Kommunikation zwischen Patienten, behandelnden Ärzten und den Zentren in den Universitäten bis hin zur Grundlagenforschung unterstützen kann, ist die Gründung einer deutschen Leberstiftung bis zum Jahr 2007 geplant. Bereits jetzt hat HepNet mit dem Förderverein „Hep-Net e.V.“ einen Grundstein für die Stiftung gelegt und sucht nun fördernde Mitglieder.

Die Fortschritte in der Therapie der Hepatitis B und C in den letzten Jahren beruhen auch auf Arbeiten des Hep-Net. So widerlegte eine bundesweite Studie bereits 2001 die Annahme, dass eine Hepatitis C eine nicht zu heilende Infektionskrankheit sei. Denn eine frühzeitige Therapie der akuten Hepatitis C kann in mehr als 90\% der Fälle eine Viruspersistenz verhindern. Kontinuierlich weiterentwickelt wurden zudem die Behandlungsstrategien: Während Mitte der 90er Jahre nur in etwa 10\% der Fälle Therapieerfolge zu verzeichnen waren, kann die chronische Hepatitis C derzeit in rund 50\% aller Fälle „geheilt" werden. Die Strategien sind dabei allerdings immer vielschichtiger geworden, sodass eine Vernetzung im Sinne eines schnellen Informationsflusses entscheidend dazu beiträgt, die Neuerungen jedem Patienten zugänglich zu machen. Ein Beispiel ist die Vorhersage eines Therapieerfolges durch die Kinetik des Virusabfalls während der Frühphase der Behandlung (Therapiewoche 12). Bei so genannten "Nonrespondern“ ist es so möglich, die Behandlung nicht unnötig lange fortzuführen. Dies reduziert nicht nur unnötige Therapiekosten, sondern auch unnötige Nebenwirkungen der Be-

handlung, die ebenfalls mit Kosten und Minderung der Lebensqualität verbunden sind. Derzeit wird unter anderem im Hep-Net geprüft, ob eine Behandlungsverkürzung und Dosisminderung bei Patienten mit den günstigen HCV-Genotypen 2 und 3 möglich ist.

Auch bei der Behandlung der chronischen Hepatitis B hat sich in den letzten Jahren viel getan. Zur individuellen Behandlung haben sich neben einer Interferontherapie Nukleos(t)idanaloga durchgesetzt, die das HepatitisB-Virus direkt hemmen. Diese neuen Medikamente können allerdings zu Resistenzen durch Mutationen des Virus führen, weshalb es notwendig werden kann, die Therapie auf andere Nukleos(t)idanaloga umzustellen. Und die Therapie mit Interferon- $\alpha$ sowohl bei der Hepatitis B als auch C ist leider mit vielen Nebenwirkungen verbunden, die oft Therapieabbrüche und Noncompliance verursachen. Da die Therapietreue aber nun einmal einer der wichtigsten Prädiktoren einer erfolgreichen Behandlung ist, gehört die Therapie mit Interferon$\alpha$ in erfahrene Hände. Fortbildungsveranstaltungen für Ärzte, adäquate medikamentöse Unterstützung und Selbsthilfegruppen für Patienten können hier einen wesentlichen Beitrag leisten, den Umgang mit Nebenwirkungen zu verbessern und damit die Compliance und den Therapieerfolg zu erhöhen.

Trotz der vielen Fortschritte bei der Behandlung der viralen Hepatitiden gibt es noch immer „Therapieversager" oder aber Patienten, die aufgrund von Kontraindikationen nicht mit diesen nebenwirkungsreichen Therapien behandelt werden dürfen. Derzeit werden daher neue Behandlungskonzepte evaluiert. Beispielsweise werden Proteaseinhibitoren oder Polymeraseinhibitoren entwickelt, die das Hepatitis-C-Virus direkt hemmen. Andere Arbeitsgruppen arbeiten an einer therapeutischen Impfung bei chronischer Hepatitis C. Bis diese Strategien in den Praxisalltag einziehen werden, werden jedoch noch einige Jahre vergehen.

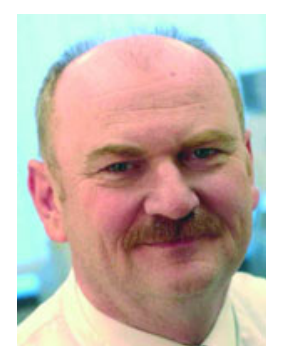

Prof. Dr. Michael P. Manns,

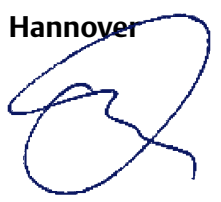

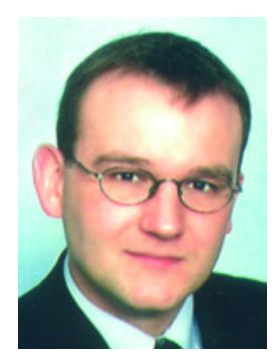

Dr. Markus Cornberg, Hannover

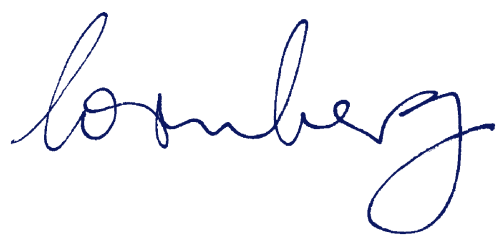

